\title{
Prevalence and Clonal Dissemination of Metallo-Beta-Lactamase-Producing Pseudomonas aeruginosa in Kermanshah
}

\author{
Alisha Akya ${ }^{1, *}$; Afsaneh Salimi ${ }^{2}$; Bizhan Nomanpour ${ }^{3}$; Kamal Ahmadi ${ }^{2}$ \\ ${ }_{1}^{1}$ Nosocomial Infection Research Centre, School of Medicine, Kermanshah University of Medical Sciences, Kermanshah, IR Iran \\ ${ }^{2}$ Student Research Committee, Faculty of Medicine, Kermanshah University of Medical Sciences, Kermanshah, IR Iran \\ ${ }^{3}$ School of Medicine, Kermanshah University of Medical Sciences, Kermanshah, IR Iran \\ ${ }^{*}$ Corresponding author: Alisha Akya, Nosocomial Infection Research Centre, School of Medicine, Kermanshah University of Medical Sciences, Kermanshah, IR Iran. Tel: \\ +98-83134274618, Fax:+98-83134262252, E-mail: akya359@yahoo.com
}

Received: January 5, 2014; Revised: August 16, 2014; Accepted: August 28, 2014

\begin{abstract}
Background: Pseudomonas aeruginosa is an opportunistic pathogen associated with nosocomial infections. The emergence and dissemination of metallo-beta-lactamases (MBLs) has contributed to the high rate of resistance among P. aeruginos $a$ isolates.

Objectives:The purpose of this study was to describe the prevalence and the clonal dissemination of MBL-producing P.aeruginosa isolates collected from major hospitals in Kermanshah.

Materials and Methods: Antibiotic susceptibility testing was performed using the minimal inhibitory concentrations. The MBLs were investigated using the Double-Disk Synergy Test (DDST) and Polymerase Chain Reaction. Molecular typing was performed by Pulsed-Field Gel Electrophoresis (PFGE).

Results: Of the 60 P. aeruginosa isolates included in this study, 30 (50\%) were resistant to Gentamicin, 38 (63.3\%) to Piperacillin, 42 (70\%) to Ceftazidime, and 45 (75\%) to Cefepime. Twenty-nine (48.3\%) isolates were MBL producers in the DDST test. Five (8.3\%) isolates were positive for the VIM gene. PFGE analysis among the MBL producers revealed 12 distinct clonal patterns.

Conclusions: The inter- and intra-hospital dissemination of resistant clones is a matter of concern and is an indicator of the level of the improvement and surveillance of standard hygiene, particularly disinfection and hand washing before and after contact with patients. Given the emergence of MBL-producing strains, surveillance has become an important procedure to control the transmission of resistant strains.
\end{abstract}

Keywords: Pseudomonas aeruginosa; Metallo-Beta-Lactamase; Clonal Dissemination; Molecular Typing

\section{Background}

Pseudomonas aeruginosa is one of the most important opportunistic pathogens among the sources of nosocomial infections, especially in burns units (1-4). Pseudomonas aeruginosa can grow in hospital environments, which are exposed to heavy antimicrobial use, and can, consequently, be transmitted rapidly among hospitalized burns patients (5). Nosocomial infections caused by $P$. aeruginosa are often difficult to treat (6). One of the major concerns for the treatment of $P$. aeruginos $a$ infections is antibiotic resistance since studies have shown that this bacterium has increasingly been resistant to many antibiotics (7, 8). Pseudomonas aeruginosa is naturally resistant to narrow-spectrum Penicillins, first- and second-generation Cephalosporins, Sulfonamides, and Trimethoprim (5). Research has shown that the prevalence of multidrug-resistant $P$. aeruginosa in burns patients is 87.5\% in Iran, $68.8 \%$ in Malaysia, and $29.24 \%$ in Pakistan (2-4). Carbapenems have been used for the treatment of multidrug-resistant $P$. aeruginosa infections. However, Carbapenem-resistant isolates have been increasing worldwide and become a major concern for public health $(9,10)$. Resistance against Carbapenems has emerged as a result of different mechanisms such as impermeability to drugs due to the loss of OprD porin, the upregulation of an active efflux pump system present in this organism, or the production of metallo-beta-lactamases (MBLs), which hydrolyze all Carbapenems $(9,11,12)$.

MBLs constitute a group of Ambler class B and have the ability to hydrolyze a wide variety of beta-lactam agents such as Penicillins, Cephalosporins, and Carbapenems. These enzymes require zinc for their catalytic activity and are inhibited by metal chelators such as ethylenediaminetetraacetic acid (EDTA) and thiol-based compounds (13). The genes encoding MBLs are typically part of an integron structure and are carried on large transferable plasmids. The MBL genes can be divided into five categories according to their molecular structures: the IMP; VIM; GIM; SIM; and SPM types (14-17). Molecular typing techniques have become important tools for the provision of information to control cross-infection and to identify the transmission pathways and sources of isolates. In addition, Pulsed-Field Gel Electrophoresis (PFGE) is now the gold standard for DNA fingerprinting techniques because of its high discriminatory power (18).

Copyright (C) 2015, Ahvaz Jundishapur University of Medical Sciences. This is an open-access article distributed under the terms of the Creative Commons Attribution-NonCommercial 4.0 International License (http://creativecommons.org/licenses/by-nc/4.0/) which permits copy and redistribute the material just in noncommercial usages, provided the original work is properly cited. 


\section{Objectives}

We aimed to determine the prevalence and clonal dissemination of MBL-producing P. aeruginosa and its antibiotic susceptibility in the isolates of major hospitals in the western Iranian city of Kermanshah.

\section{Materials and Methods}

\subsection{Bacterial Isolates}

Sixty isolates of P. aeruginosa were collected from two hospitals in Kermanshah (Imam Khomeini [A] and Imam Reza [B]) between 2011 and 2012. The specimens were collected from burns ( $n=32,53.3 \%)$, urine ( $n=9,15 \%)$, respiratory tract secretions ( $\mathrm{n}=10,16.7 \%)$, and other sources (vagina, catheter, eye, ear, wound, and blood) $(n=9,15 \%)$. The bacteria were then cultured on the MacConkey agar and the Muller-Hinton agar media, and the grown colonies were confirmed using specific tests. These included catalase, oxidase, the growth characteristics and pigment gas production, indole and methyl red, Sulfide Indole Motility, and growth at $42^{\circ} \mathrm{C}$ on the Cetrimide agar (Merck, Germany).

\subsection{Antibiotic Susceptibility Testing}

Antibiotic susceptibility testing was performed as recommended by The Clinical and Laboratory Standards Institute (CLSI). Minimal inhibitory concentrations for 4 antibiotics, namely Gentamicin, Piperacillin, Ceftazidime, and Cefepime (MAST, England), were determined using the Micro-Broth Dilution method (19).

\subsection{Identification of Metallo-Beta-Lactamases}

The Double-Disk Synergy Test (DDST) was used to screen the MBL-producing P. aeruginosa isolates (15). Disks containing $930 \mu \mathrm{g}$ of EDTA (SinaClon, Iran) plus $10 \mu \mathrm{g}$ of Imipenem (SinaClon, Iran) were placed on the Muller Hinton agar spread by isolates. An increase of $\geq 7 \mathrm{~mm}$ in the inhibited zone diameter in the presence of $930 \mu \mathrm{g}$ of EDTA compared to Imipenem tested alone was considered to be a positive test for the presence of MBLs. For the molecular diagnostic of the MBLs, the boiling method was used for DNA extraction (20). Polymerase Chain Reaction (PCR) was carried out using Master Mix (SinaClon, Iran), DNA, and a pair of primers (SinaClon, Iran). The primers used for the amplification of the VIM were VIMF (5'-GTT TGG TCG CAT ATC GCA AC-3) and VIMR (5-AAT GCG CAG CAC CAG GAT AG3), which amplified a 382-bp DNA fragment (15). The PCR product was determined using gel electrophoresis ( $1 \%$ agarose) and staining with ethidium bromide, followed by visualization using a Gel Doc apparatus (Bio-Rad, USA).

\subsection{Pulsed-Field Gel Electrophoresis}

PFGE was performed according to a previously described protocol (21) with some modifications. The MBLproducing $P$. aeruginosa plugs were digested with $20 \mathrm{U}$ SpeI (Fermentas). Salmonella enterica serovar Braenderup
H9812 was digested with $20 \mathrm{U}$ XbaI (Fermentas) and used as an electrophoresis molecular weight marker. Following SpeI digestion, the plugs were loaded into a $1 \%$ lowelectroendosmosis agarose (Merck, Germany). Electrophoresis was performed on a CHEF Mapper apparatus (Bio-Rad, USA) at $14^{\circ} \mathrm{C}$ for 22 hours under the following conditions: initial switch time of 5 seconds; final switch time of 35 seconds; included angle of $120^{\circ} \mathrm{C}$; voltage gradient of $6 \mathrm{~V} / \mathrm{cm}$; and linear ramping factor. The gels were stained with ethidium bromide and visualized under UV light using a Gel Doc apparatus (Bio-Rad, USA).

\subsection{Software Analysis}

DNA fragment analysis was performed using GelCompar II (version 6.6) (Applied Maths, Belgium). The Dice coefficient was used to calculate similarities, and the Unweighted Pair Group Method with Arithmetic Mean (UPGMA) was employed for cluster analysis. A cluster of isolates was defined to include all isolates with more than $80 \%$ similarity in their DNA patterns according to the Tenover criteria $(22,23)$.

\subsection{Statistical Analysis}

The statistical analysis of the data was done using the Statistical Package for the Social Sciences (SPSS) (version 16). The chi-square test was utilized to assess the correlation between the data. Statistical significance was defined as a $P$ value $<0.05$.

\section{Results}

\subsection{Bacterial Isolates}

Of the 60 P. aeruginosa isolates, 47 were from hospital A and 13 from hospital B. The specimens were collected form 31 (51.7\%) males and 29 (48.3\%) females at an average age of $31.64 \pm 23.97$ and $28.66 \pm 19.90$ years old, respectively.

\subsection{Antibiotic Susceptibility of Isolates}

Of the $60 \mathrm{P}$. aeruginosa isolates, 30 (50\%) were resistant to Gentamicin, 38 (63.3\%) to Piperacillin, 42 (70\%) to Ceftazidime, and 45 (75\%) to Cefepime. The highest resistance to the 4 antibiotics belonged to the isolates from the burns specimens (Table 1 ).

\subsection{Screening for Metallo-Beta-Lactamase by Double-Disk Synergy Test and Polymerase Chain Reaction}

The DDST test determined that 29 (48.3\%) isolates were MBL producers. Of this total, 5 (8.3\%) strains were positive for the VIM gene (Figure 1) and 4 were from the burns specimens. Of the VIM-producing isolates, 4 (80\%) were resistant to Cefepime, Ceftazidime, and Piperacillin. The data analysis via the chi-square test showed no significant association between resistance to antibiotics and MBLs $(\mathrm{P}=0.5)$. 
Akya A et al.

Table 1. The Source and Antibiotic Resistance of the P. aeruginosa Isolates

\begin{tabular}{lcccc}
\hline Sample Source & \multicolumn{4}{c}{ Antibiotic Resistance, \% } \\
\cline { 2 - 5 } & Cefepime & Ceftazidime & Piperacilin & Gentamicin \\
\hline Burns & 90.6 & 84.4 & 78.1 & 56.2 \\
Urine & 44.4 & 33.3 & 44.4 & 22.2 \\
Respiratory Tract & 60 & 60 & 40 & 50 \\
Other & 66.7 & 66.7 & 55.6 & 55.6 \\
Total & 75 & 70 & 63.3 & 50 \\
\hline
\end{tabular}

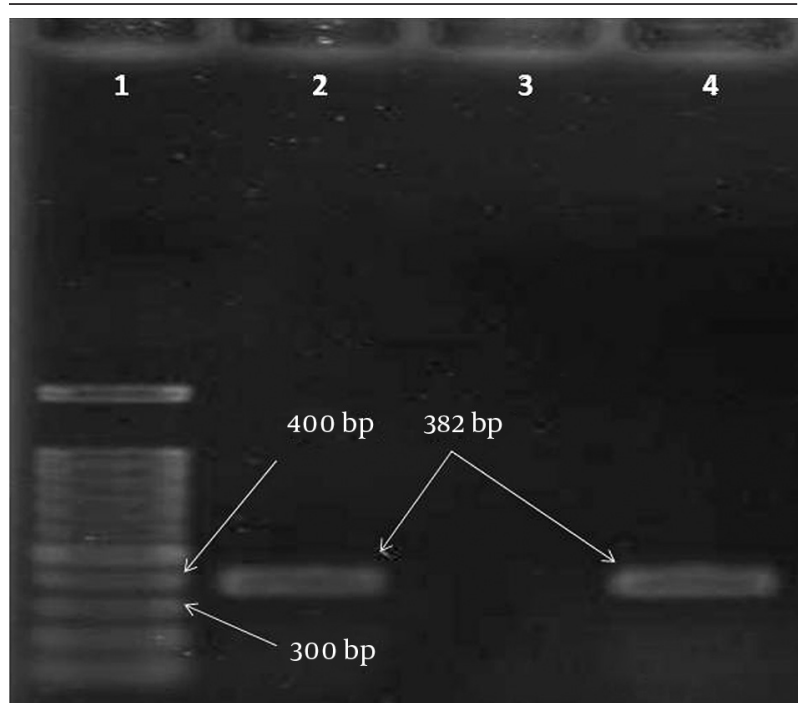

Figure 1. Electrophoresis of Polymerase Chain Reaction Products for the blaVIM Gene among the Pseudomonas aeruginosa Isolates

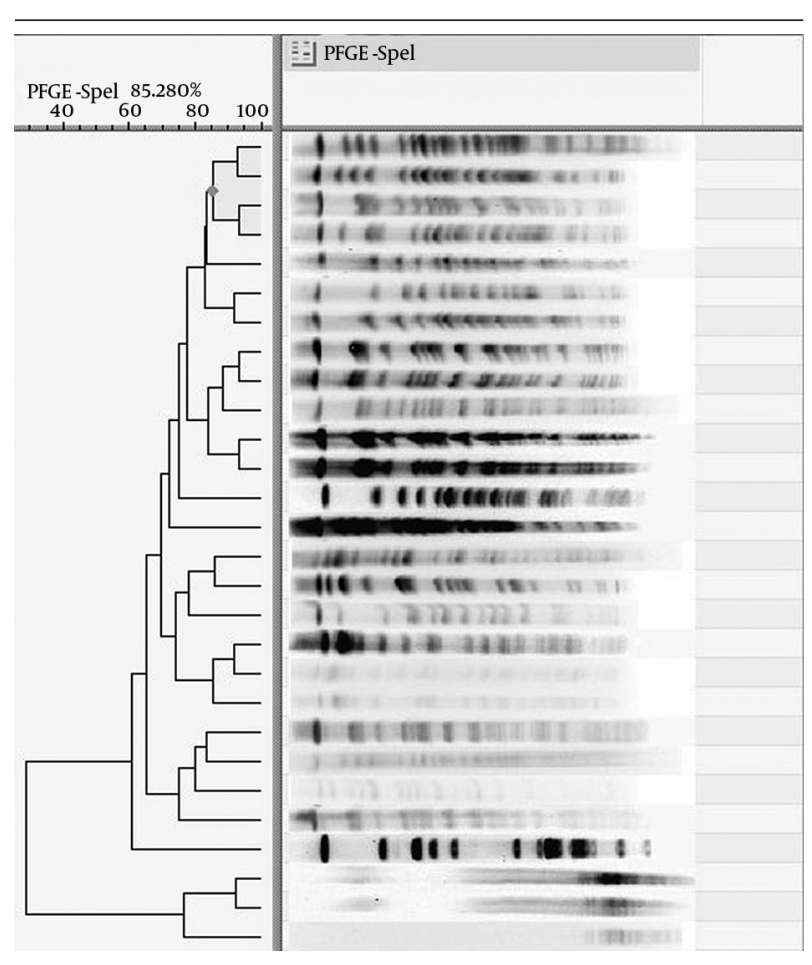

Figure 2. Dendrogram Based on the Pulsed Field Gel Electrophoresis Patterns of the Pseudomonas aeruginosa Isolates

\subsection{Pulsed-Field Gel Electrophoresis}

The clonal relationship among the MBL producers revealed 12 patterns: $A(n=7) ; B(n=5) ; C(n=1) ; D(n=1)$; $\mathrm{E}(\mathrm{n}=2) ; \mathrm{F}(\mathrm{n}=1) ; \mathrm{G}(\mathrm{n}=3) ; \mathrm{H}(\mathrm{n}=3) ; \mathrm{I}(\mathrm{n}=1) ; \mathrm{J}(\mathrm{n}=1) ; \mathrm{K}(\mathrm{n}$ $=2)$; and $\mathrm{L}(\mathrm{n}=1)$ (Figure 2$)$. Among them, one strain was untypeable by PFGE and 6 strains had unique patterns. The pattern having the highest number of strains was defined as the dominant clone. The strains of this clone were from a variety of specimens obtained from the two hospitals in this study.

\section{Discussion}

A major challenge concerning P. aeruginosa infections is the ability of this bacterium to acquire resistance against new antibiotics (24). Our results showed that the isolates collected for the purposes of the present study were resistant against a variety of antibiotics. It seems that the inappropriate prescription of antibiotics in our area is one of the reasons for the increasing antibiotic resistance of this opportunistic pathogen. Furthermore, thanks to its genetic nature, $P$. aeruginosa is capable of acquiring a wide range of genes by means of plasmids and transposons, which allow this bacterium to become rapidly resistant against the different classes of antibiotics. The production of MBLs is one of the main mechanisms of resistance against antibiotics among $P$. aeruginosa isolates. Indeed, the emergence of MBL-producing P. aeruginosa isolates in hospital environments is a serious problem for the management of nosocomial infections (21). As MBL genes are located on transferable elements such as plasmids and integrons, they can easily disseminate between the strains of the bacterium.

According to recent studies in Japan, Italy, and Columbia, even without using antibiotics, P. aeruginosa isolates were capable of acquiring the VIM and IMP genes (25-27). The strains that carried these genes were also resistant to other classes of antibiotics such as Quinolones, Sulfonamides, and Aminoglycosides (28). According to epidemiological studies, the prevalence of the various patterns of drug resistance, especially the genes encoding the MBLs, can be different among $P$. aeruginosa isolates from one region to another or even between different hospitals in the same area (13). In Asian countries, the rates of MBL production by $P$. aeruginosa have been reported as $6.2 \%$ in Korea, $26.6 \%$ in Japan, and 47.3\% in Taiwan (12, 29, 30). Also, studies in several parts of the world have demonstrated various rates of 
MBL production by P. aeruginosa, including 38.3\% in Brazil, $35 \%$ in Canada, and $62 \%$ in Greece $(21,31,32)$.

In our study, almost half of the isolates produced MBL enzymes, which is consistent with the above-mentioned results. Some studies in Iran have reported higher rates of MBL production among P. aeruginosa isolates (e.g. $55.8 \%$ in Isfahan and $72 \%$ in Tehran) $(20,33)$. These differences can be explained by the geographic areas, prescribed antibiotic patterns, and the type and number of the samples tested. Among several genes encoding the MBLs, the VIM gene in $P$. aeruginosa is the most common (15). However, previous research has demonstrated that the frequency of this gene among $P$. aeruginosa isolates in Iran is low in comparison to other countries such as South Korea, Japan, and Canada (15, 33-35). In our study, the prevalence of the VIM gene was $8.3 \%$, which is similar to the results of the previous research in Iran. Therefore, in our area, other resistant genes or mechanisms may be involved such as class D Carbapenemases, efflux pumps, and defects and loss of outer-membrane proteins including OprD (36).

The main sources of the acquisition of $P$. aeruginosa infections among patients have been investigated in different parts of world. Some studies have suggested that $P$. aeruginosa spreads from patient to patient (37), while other studies have indicated that this pathogen is predominantly acquired from the environment (38). In our study, the majority of the $P$. aeruginosa isolates in dominant clone A, with the highest number of strains, were from the lung specimens in both hospitals. There were strains from burn, urine, and catheter specimens in this clone as well. Given the high similarity between the genomic patterns of these strains in the current study, it can be concluded that the strains may have disseminated among the wards of each hospital and even between the two hospitals. In clone E, there were 2 strains, one from hospital A and the other from hospital B, indicating that these strains originated from a common source. Furthermore, there were 2 strains of $P$. aeruginosa with unique genomic patterns susceptible to Gentamicin, Piperacillin, Ceftazidime, and Cefepime which were isolated from the urine and eye specimens: they may have originated from environmental sources. The strains of clones B, C, D, E, H, I, and L were resistant to the third and fourth generations of Cephalosporins; they may have originated from a common source. The other strains, which had unique genomic patterns or contained the VIM gene, may have disseminated amongst the patients in the two hospitals from different sources.

In conclusions, our findings showed that the P. aeruginosa isolates tested were from deferent clonal origins. While some of the $P$. aeruginosa strains may have originated from environmental sources, the others may have disseminated among the patients in the two hospitals under study by various ways of transmission. The interand intra-hospital dissemination of resistant clones is a great concern and is an indicator of the level of the im- provement and surveillance of standard hygiene procedures, not least disinfection and hand washing before and after contact with patients. According to our results, the resistance rate of $P$. aeruginosa is high and preventive strategies such as more precise antibiotic selection for treatment and less physical contact between patients should be adopted. Given the clinical significance of MBLproducing isolates, the identification of these organisms is critical if hospitals are to offer a more optimal therapeutic response and control of bacterial dissemination.

\section{Acknowledgements}

This paper was derived from an MSc thesis on medical microbiology. We thank Kermanshah University of Medical Sciences for its financial support of this work.

\section{Authors' Contributions}

Alisha Akya carried out the design and coordinated the study, participated in most of the experiments, and prepared the manuscript. Afsaneh Salimi provided assistance in the design of the study, coordinated and carried out all the experiments, and participated in manuscript preparation. Bizhan Nomanpour and Kamal Ahmadi provided assistance for all the experiments. All the authors have read and approved the content of the manuscript.

\section{Funding/Support}

Kermanshah University of Medical Sciences financially supported this work.

\section{References}

1. Mahzounieh M, Khoshnood S, Ebrahimi A, Habibian S, Yaghoubian M. Detection of Antiseptic-Resistance Genes in Pseudomonas and Acinetobacter spp. Isolated From Burn Patients. Jundishapur J Nat Pharm Prod. 2014;9(2):e15402.

2. Lim KT, Yasin RM, Yeo CC, Puthucheary SD, Balan G, Maning N, et al. Genetic fingerprinting and antimicrobial susceptibility profiles of Pseudomonas aeruginosa hospital isolates in Malaysia. $J$ Microbiol Immunol Infect. 2009;42(3):197-209.

3. Mirsalehian A, Feizabadi M, Nakhjavani FA, Jabalameli F, Goli H, Kalantari N. Detection of VEB-1, OXA-10 and PER-1 genotypes in extended-spectrum $\beta$-lactamase-producing Pseudomonas aeruginosa strains isolated from burn patients. Burns. 2010;36(1):70-4.

4. Ullah F, Malik SA, Ahmed J. Antimicrobial susceptibility and ESBL prevalence in Pseudomonas aeruginosa isolated from burn patients in the North West of Pakistan. Burns. 2009;35(7):1020-5.

5. Lambert PA. Mechanisms of antibiotic resistance in Pseudomonas aeruginosa.J Royal Soc Med. 2002;95(Suppl 41):22.

6. Xiao H, Ye X, Liu Q, Li L. Antibiotic susceptibility and genotype patterns of from mechanical ventilation-associated pneumonia in intensive care units. Biomed Rep. 2013;1(4):589-93.

7. Strateva T, Yordanov D. Pseudomonas aeruginosa-a phenomenon of bacterial resistance. JMed Microbiol. 2009;58(9):1133-48.

8. Cholley P, Gbaguidi-Haore H, Bertrand X, Thouverez M, Plésiat P, Hocquet D, et al. Molecular epidemiology of multidrug-resistant Pseudomonas aeruginosa in a French university hospital. J Hospital Infect. 2010;76(4):316-9.

9. Wanq J, Zhou JY, Qu TT, Shen P, Wei ZQ, Yu YS. Molecular epidemiology and mechanisms of carbapenem resistance in Pseudomonas aeruginosa isolates from Chinese hospitals. IJAA. 2010;35(5):486-91.

10. Yoo JS, Yang JW, Kim HM, Byeon J, Kim HS, Yoo JI, et al. Dissemina- 
tion of genetically related IMP-6-producing multidrug-resistant Pseudomonas aeruginosa ST235 in South Korea. Int J Antimicrob Agents. 2012;39(4):300-4.

11. Köhler T, Michea-Hamzehpour M, Epp SF, Pechere J. Carbapenem activities against Pseudomonas aeruginosa: respective contributions of OprD and efflux systems. Antimicrob Agents Chemother. 1999;43(2):424-7.

12. Kouda S, Ohara M, Onodera M, Fujiue Y, Sasaki M, Kohara T, et al Increased prevalence and clonal dissemination of multidrug-resistant Pseudomonas aeruginosa with the blaIMP-1 gene cassette in Hiroshima.J Antimicrobi Chemother. 2009:dkp142.

13. Walsh TR, Toleman MA, Poirel L, Nordmann P. Metallo-betalactamases: the quiet before the storm? Clin Microbiol Rev. 2005;18(2):306-25.

14. Valenza G, Joseph B, Elias J, Claus H, Oesterlein A, Engelhardt $\mathrm{K}$, et al. First survey of metallo- $\beta$-lactamases in clinical isolates of Pseudomonas aeruginosa in a German university hospital. Antimicrob Agents Chemother. 2010;54(8):3493-7.

15. Pitout JDD, Gregson DB, Poirel L, McClure J, Le P, Church DL. Detection of Pseudomonas aeruginosa producing metallo- $\beta$ lactamases in a large centralized laboratory. J Clin Microbiol. 2005;43(7):3129-35.

16. Lauretti L, Riccio ML, Mazzariol A, Cornaglia G, Amicosante G, Fontana R, et al. Cloning and characterization of blaVIM, a new integron-borne metallo-beta-lactamase gene from a Pseudomonas aeruginosa clinical isolate. Antimicrob Agents Chemother. 1999;43(7):1584-90.

17. Castanheira M, Toleman MA, Jones RN, Schmidt FJ, Walsh TR. Molecular characterization of a beta-lactamase gene, blaGIM-1, encoding a new subclass of metallo-beta-lactamase. Antimicrob Agents Chemother. 2004;48(12):4654-61.

18. Kidd TJ, Grimwood K, Ramsay KA, Rainey PB, Bell SC. Comparison of three molecular techniques for typing Pseudomonas aeruginosa isolates in sputum samples from patients with cystic fibrosis. J Clin Microbiol. 2011;49(1):263-8.

19. Clinical and Laboratory Standards Institute. Performance standards for antimicrobial susceptibility testing. Twenty-First informational supplement. Wayne: 2011.

20. Fazeli H, Moslehi Z, Irajian G, Salehi M. Determination of Drug resistance patterns and detection of bla-VIM gene in Pseudomonas aeruginosastrains Isolated from burned patients in the Emam Mosa Kazem hospital, Esfahan, Iran (2008-9). Iran JMed Microbiol. 2010;3(4):1-8.

21. Pitout JD, Chow BL, Gregson DB, Laupland KB, Elsayed S, Church DL. Molecular epidemiology of metallo-beta-lactamase-producing Pseudomonas aeruginosa in the Calgary Health Region: emergence of VIM-2-producing isolates. J Clin Microbiol. 2007;45(2):294-8.

22. Tsutsui A, Suzuki S, Yamane K, Matsui M, Konda T, Marui E, et al. Genotypes and infection sites in an outbreak of multidrug-resistant Pseudomonas aeruginosa. J Hospital Infect. 2011;78(4):317-22.

23. Tenover FC, Arbeit RD, Goering RV, Mickelsen PA, Murray BE, Persing $\mathrm{DH}$, et al. Interpreting chromosomal DNA restriction patterns produced by pulsed-field gel electrophoresis: criteria for bacterial strain typing. J Clin Microbiol. 1995;33(9):2233-9.

24. Mohan BS, Lava R, Prashanth HV, Vinod Nambiar MB, Nayak Venkatesh R, Baragundi Mahesh SR. Prevalence and antibiotic sensitivity pattern of pseudomonas aeruginosa; an emerging nosoco- mial pathogen. Int J Biol Med Res. 2013;4(4):2729-31.

25. Cornaglia G, Mazzariol A, Lauretti L, Rossolini GM, Fontana R. Hospital outbreak of carbapenem-resistant Pseudomonas aeruginosa producing VIM-1, a novel transferable metallo-beta-lactamase. Clin Infect Dis. 2000;31(5):1119-25.

26. Yatsuyanagi J, Saito S, Harata S, Suzuki N, Ito Y, Amano K, et al. Class 1 integron containing metallo-beta-lactamase gene blaVIM-2 in Pseudomonas aeruginosa clinical strains isolated in Japan. Antimicrob Agents Chemother. 2004;48(2):626-8.

27. Crespo MP, Woodford N, Sinclair A, Kaufmann ME, Turton J, Glover J, et al. Outbreak of carbapenem-resistant Pseudomonas aeruginosa producing VIM-8, a novel metallo-beta-lactamase, in a tertiary care center in Cali, Colombia. J Clin Microbiol. 2004;42(11):5094-101.

28. Nordmann P, Poirel L. Emerging carbapenemases in Gram negative aerobes. Clin Microbiol Infect. 2002;8(6):321-31.

29. Yong D, Choi YS, Roh KH, Kim CK, Park YH, Yum JH, et al. Increasing prevalence and diversity of metallo-beta-lactamases in Pseudomonas spp., Acinetobacter spp., and Enterobacteriaceae from Korea. Antimicrob Agents Chemother. 2006;50(5):1884-6.

30. Lee M, Peng C, Hsu H, Chen Y. Molecular characterisation of the metallo- $\beta$-lactamase genes in imipenem-resistant Gram-negative bacteria from a university hospital in southern Taiwan. Int $J$ Antimicrob Agents. 2008;32(6):475-80.

31. Roberto ML, Sirlei GM, Luís HB, Afonso GA, Silvio G, Azizedite GG Phenotypic detection of metallo-ß-lactamases in Pseudomonas aeruginosa and Acinetobacter baumannii isolated from hospitalized patients in São Luis, State of Maranhão, Brazil. SBMT. 2013;46(4):506-9.

32. Giakkoupi P, Petrikkos G, Tzouvelekis LS, Tsonas S, Legakis NJ, Vatopoulos AC, et al. Spread of integron-associated VIM-type metallo-beta-lactamase genes among imipenem-nonsusceptible Pseudomonas aeruginosa strains in Greek hospitals. J Clin Microbiol. 2003;41(2):822-5.

33. Shahcheraghi F, Nikbin VS, Feizabadi MM. Identification and genetic characterization of metallo-beta-lactamase-producing strains of Pseudomonas aeruginosa in Tehran, Iran. New Microbiol. 2010;33(3):243-8.

34. Shibata N, Doi Y, Yamane K, Yagi T, Kurokawa H, Shibayama K, et al. PCR typing of genetic determinants for metallo-beta-lactamases and integrases carried by gram-negative bacteria isolated in Japan, with focus on the class 3 integron. J Clin Microbiol. 2003;41(12):5407-13.

35. Lee K, Park A, Kim M, Lee H, Cho J, Kang J, et al. Metallo- $\beta$ lactamase-producing Pseudomonas spp. in Korea: high prevalence of isolates with VIM-2 type and emergence of isolates with IMP-1 type. Yonsei Med J. 2009;50(3):335-9.

36. Rodríguez-Martínez J, Poirel L, Nordmann P. Molecular epidemiology and mechanisms of carbapenem resistance in Pseudomonas aeruginosa. Antimicrob Agents Chemother. 2009;53(11):4783-8.

37. Tummler B, Koopmann U, Grothues D, Weissbrodt H, Steinkamp $G$, von der Hardt H. Nosocomial acquisition of Pseudomonas aeruginosa by cystic fibrosis patients. J Clin Microbiol. 1991;29(6):1265-7.

38. Speert DP, Campbell ME, Henry DA, Milner R, Taha F, Gravelle A, et al. Epidemiology of Pseudomonas aeruginosa in cystic fibrosis in British Columbia, Canada. Am J Respir Crit Care Med. 2002;166(7):988-93. 